\section{Prevention of serious infections in hereditary hemorrhagic telangiectasia: roles for prophylactic antibiotics, the pulmonary capillaries- but not vaccination}

Rodríguez-García et al. recently suggested that vaccination against Haemophilus influenzae, streptococcus pneumonia, and influenza should be considered a potential route to prevent the major deep-seated infections observed in some patients with hereditary hemorrhagic telangiectasia $\left(\mathrm{HHT}^{1}\right)$. However, as pointed out by Al-Samkari et al., ${ }^{2}$ there is no current evidence that patients with HHT are more prone to pneumoccal, hemophilus or influenza infections, or would benefit from any different approach to vaccination than the general population. Furthermore, we are concerned that a focus on these pathogens risks missing the relevant necessary foci that require a different approach- transient bacteremias, prophylactic antibiotics, and pulmonary capillary clearance functions.

HHT is caused by pathogenic variants in ENG, $A C V R L 1$, and SMAD4, and usually results in vascular abnormalities (telangiectasia, arteriovenous malformations (AVMs)), recurrent bleeds, and anemia. ${ }^{3}$ A subgroup of HHT patients develop occasional deep-seated infections including brain abscesses. ${ }^{4-6}$ HHT members of the European Reference Network for Rare Vascular Diseases (VASCERN) have contributed strongly to published primary data, including recent manuscripts detailing clinical and microbiological features of $65^{4}$ and $27^{5}$ consecutive brain abscess cases. Multidisciplinary discussions on optimal prevention strategies have been taking place with our microbiological colleagues for more than a decade. Additionally, in June 2017, a VASCERN HHT workshop specifically addressed infection in HHT, with proceedings published earlier this year. ${ }^{8}$ The workshop was prompted both by considerations on the infections, ${ }^{3-7}$ and the 2016 publication of a manuscript demonstrating modified responses by macrophages from a myeloid-restricted murine model of HHT (Eng $g^{f / f l}$ LysMCre mice). ${ }^{9}$

Key microbiological considerations are:

(i) Brain abscesses and other deep-seated spinal, joint and other infections occur at increased frequency in HHT patients, but the etiological organisms (summarised in ${ }^{4-6}$ ) are quite different to those causing vaccine-preventable invasive infections. In fact, these severe HHT infections are caused by heterogenous flora, often mixed, including anerobic and aerobic commensals of the gastrointestinal and periodontal spaces. For example, at least seventeen different species were identified in the twenty-four positive cerebral abscess isolates, ${ }^{4}$ and none would have been expected to be responsive to the stated vaccines.

Isolated species were Streptococcus Milleri group; Streptococcus anginosus; Non-hemolytic streptococci; alpha hemolytic streptococci; uncharacterised streptococci, Actinomycosis spp; Staphylococcus intermedius; unspecified anaerobe species; Bacteroides spp.; Propionobacteria; Porphyromonas, Gemella; Peptostreptococcus; enterococcus; uncharacterised Gram positive rods and Gram positive cocci, and only in one case, a species for which some work on vaccines is in progress (methicillin resistant staphylococcus aureus $(M R S A))^{4}$. There is thus no current possibility of vaccinating against these organisms.

(ii) Rodríguez-García et al. ${ }^{1}$ did not discuss that transient bacteremia (viable organisms circulating in blood) is very frequent after dental and other invasive procedures in the general population. ${ }^{10}$ Positive blood cultures increased from $4-9 \%$ to $96 \%$ within 30 seconds of a tooth extraction. ${ }^{10}$ Species isolated from 421 positive cul- tures $^{10}$ overlapped with those cultured from HHT abscesses $^{4-6}$ but did not include Streptococcus pneumoniae or Haemophilus influenzae. Such concepts are well known in microbiological circles, but less appreciated within mainstream medical and surgical disciplines. How such bacteria translocate from the periodontal region to be pathogenic at distal sites does not seem to be a prominent area of active research.

(iii) The reason why positive blood cultures post dental extraction fall rapidly (to $65 \%$ at 15 minutes, $18 \%$ at 1 hour ${ }^{10}$ ) is not fully understood, and commonly attributed to clearance by the "reticuloendothelial system". Patients with HHT provide a more specific clue, because it is the subgroup with pulmonary AVMs who are predominantly affected by deep-seated infections from periodontal and other micro-organisms. Pulmonary AVMs provide a right-to-left shunt, enabling bacteria in venous blood to bypass the pulmonary circulation. Whether the bypassed mechanism is a simple mechanical filter of bacterial-cellular/embolic aggregates, or in close vicinity to pulmonary capillary macrophages, is not yet known, but the implication is that the normal pulmonary capillary bed is a major, if not the major site of removal of such bacteria. This is one of the reasons for pulmonary AVM screening programmes, and why embolization treatment is recommended for all pulmonary AVMs where feasible, irrespective of symptoms. ${ }^{2,3}$

(iv) Limeres Posse et al. clearly demonstrated that bacteremias post dental extraction can be prevented by appropriate antibiotic prophylaxis. ${ }^{10}$ Prophylactic antibiotics prior to dental and surgical procedures has been a longstanding recommendation for patients with HHT and pulmonary AVMs, ${ }^{3-6}$ and this is one of the five VASCERN HHT Outcome Measures published in $2018 .{ }^{3}$

Currently unsolved questions include:

- Whether the murine data ${ }^{9}$ imply that all patients with ENG HHT (or indeed all HHT patients) should also have prophylactic antibiotics prior to bacteremia-precipitating interventions.

- Whether the right-to-left shunting by more common states such as patent foramen ovale also increases risks of deep-seated infections from transient bacteremias: notably, brain abscess is also a complication of congenital heart disease, attributed to right-to-left shunting.

- How best to prevent infection for at risk patients: local options for instance, have included long term penicillin as for post-splenectomy patients.

- How to optimise iron levels given bacterial infections are more frequent and severe in the setting of high iron levels, which counterintuitively, can occur transiently after iron treatments.

We agree with Al-Samkari et al., ${ }^{2}$ that patients with HHT should have the usual vaccination strategies, as for members of the general population. In most countries, this includes pneumococcal and haemophilus influenza type $b$ which can cause invasive disease and meningitis in any individual - but there is no evidence that HHT patients are at any more or less risk of these infections than the general population. They are however at a significantly increased risk of polymicrobial, deep-seated infections and abscesses especially if they have pulmonary AVMs. Reducing risks of the abscesses and other infections associated with pulmonary AVMs in HHT patients will require a more fundamental understanding of transient dental and surgical bacteremias, with a focus on pulmonary capillary clearance functions. It is important that the question of vaccination, which should be a normal part of infection prevention for everyone, does 
not obscure the need for a better focus on how to prevent these bacteremias from causing life altering, deep-seated infections.

Claire Shovlin, ${ }^{1}$ Kathleen Bamford, ${ }^{2}$ Carlo Sabbà, ${ }^{3}$ Hans-Jurgen Mager, ${ }^{4}$ Anette Kjeldsen, ${ }^{5}$ Freya Droege, ${ }^{6}$ Elisabetta Buscarini and Sophie Dupuis-Girod ${ }^{7}$ on behalf of VASCERN HHT

${ }^{1}$ Respiratory Medicine, and VASCERN HHT European Reference Centre, Hammersmith Hospital, Imperial College Healthcare NHS Trust, London; NHLI Vascular Science, Imperial College London, UK; ${ }^{2}$ Department of Microbiology and VASCERN HHT European Reference Centre, Hammersmith Hospital, Imperial College Healthcare NHS Trust, London, UK; ${ }^{3}$ Center for Rare Diseases, "Frugoni" Internal Medicine Unit, Interdepartmental HHT Center, Interdisciplinary Department of Medicine and VASCERN HHT European Reference Centre, University of Bari School of Medicine, Italy; ${ }^{4}$ Department of Pulmonology and VASCERN HHT European Reference Centre, St. Antonius Hospital, Koekoekslaan 1, 3435 CM Nieuwegein, the Netherlands; 'Department of Otorhinolaryngology and VASCERN HHT European Reference Centre, Odense University Hospital, University of Southern Denmark, Denmark; ' ${ }^{\circ}$ epartment of Otorhinolaryngology and VASCERN HHT European Reference Centre, Essen University Hospital, Germany; ${ }^{7}$ Gastroenterology Department and VASCERN HHT European Reference Centre, Maggiore Hospital, ASST Crema, Italy and ${ }^{8}$ Hospices Civils de Lyon, Hôpital Femme-Mère-Enfants, Service de Génétique, and VASCERN HHT European Reference Centrel centre de Référence pour la maladie de Rendu-Osler, France, and Université de Lyon, Faculté de médecine, France

Correspondence:c.shovlin@imperial.ac.uk doi:10.3324/haematol.2018.209791

Information on authorship, contributions, and financial \& other disclosures was provided by the authors and is available with the online version of this article at www. haematologica.org.

\section{References}

1. Rodríguez-García J, Zarrabeitia-Puente R, Fernández-Santos R, García-Erce JA. Infection prevention in patients with hereditary hemorrhagic telangiectasia. Haematologica. 2018;103(10):e491-e492.

2. Al-Samkari $\mathrm{H}$, Kritharis $\mathrm{A}$, Kuter DJ. Infections and vaccination in hereditary hemorrhagic telangiectasia: microbiological evidencebased considerations. Haematologica. 2018;103(10):e492-e495.

3. Shovlin CL, Buscarini E, Kjeldsen AD, et al. European Reference Network For Rare Vascular Diseases (VASCERN) outcome measures for hereditary haemorrhagic telangiectasia (HHT). Orphanet J Rare Dis. 2018;13(1):136.

4. Boother EJ, Brownlow S, Tighe HC, Bamford KB, Jackson JE, Shovlin CL. Cerebral abscess associated with odontogenic bacteremias, hypoxemia, and iron loading in immunocompetent patients with right-to-left shunting through pulmonary arteriovenous malformations. Clin Infect Dis. 2017;65(4):595-603.

5. Mathis S, Dupuis-Girod S, Plauchu H, et al. Cerebral abscesses in hereditary haemorrhagic telangiectasia: a clinical and microbiological evaluation. Clin Neurol Neurosurg. 2012;114(3):235-240.

6. Dupuis-Girod S, Giraud S, Decullier E, et al. Hemorrhagic hereditary telangiectasia (Rendu-Osler disease) and infectious diseases: an underestimated association. Clin Infect Dis. 2007;44(6):841-845.

7. Shovlin C, Bamford K, Wray D. Post-NICE 2008: Antibiotic prophylaxis prior to dental procedures for patients with pulmonary arteriovenous malformations (PAVMs) and hereditary haemorrhagic telangiectasia. Br Dent J. 2008;205(10):531-533.

8. Shovlin CL and Botella LM. VASCERN HHT Workshop on Immunity, injury, and inflammation in HHT and HHT vessels, Dubrovnik, Croatia, June 2017: Available at https://vascern.eu/wpcontent/uploads/2018/03/Immunity-and-Inflammation-Workshop2017.pdf

9. Ojeda-Fernandez L, Recio-Poveda L, Aristorena M, et al. Mice lacking endoglin in macrophages show an impaired immune response. PLoS Genet. 2016:12(3):e1005935.

10. Limeres Posse J, Alvarez Fernandez M, Fernandez Feijoo J, et al Intravenous amoxicillin/clavulanate for the prevention of bacteraemia following dental procedures: a randomized clinical trial. J Antimicrob Chemother. 2016;71:2022-2030. 